Advances in Science, Technology and Engineering Systems Journal Vol. 5, No. 5, 1233-1246 (2020)

\title{
Design and Development of Electronic Sensor and Monitoring System of Smart Low-cost Phototherapy Light System for Non-Invasive Monitoring and Treatment of Neonatal Jaundice
}

\author{
Paul Cabacungan ${ }^{1, *}$, Carlos Oppus ${ }^{1,2}$, Gregory Tangonan ${ }^{1}$, Nerissa Cabacungan ${ }^{3}$, John Paul Mamaradlo ${ }^{1}$, Neil Angelo Mercado ${ }^{1}$ \\ ${ }^{1}$ Ateneo Innovation Center, Ateneo de Manila University, Quezon City, 1108, Philippines \\ ${ }^{2}$ Electronics, Computer, and Communications Engineering Department, Ateneo de Manila University, Quezon City, 1108, Philippines
}

${ }^{3}$ Ateneo Grade School, Ateneo de Manila University, Quezon City, 1108, Philippines

A R T I C L E I N F O

Article history:

Received: 31 August, 2020

Accepted: 28 September, 2020

Online: 24 October, 2020

Keywords:

Phototherapy

Neonatal Jaundice

Yellow Detection

Irradiance

Sensor and Monitoring System

\begin{abstract}
A B S T R A C T
This paper showcases our previous and continuously improving development at Ateneo Innovation Center (AIC) and partners in designing and further enhancing the existing Lowcost Phototherapy Light System (LPLS) and Improved Low-cost Phototherapy Light System (ILPLS) to the new Smart Low-cost Phototherapy Light System (Smart LPLS) with noninvasive jaundice monitoring for newborns with Neonatal Jaundice (NNJ). Developing this tool will help determine the intensity of yellowish color in infants and can monitor NNJ in a non-invasive way. The system is envisioned to be integrated with Mobile or Near Cloud as part of Smart Nursing Station together with other hospital equipment for monitoring, collection, and management of medical records and services. Its solar-power features for off-grid and remote deployments were also explored. This contribution is an extension of the Intelligent Sensors and Monitoring System for Low-cost Phototherapy Light for Jaundice Treatment that was presented in the International Symposium on Multimedia and Communication Technology (ISMAC) in 2019.
\end{abstract}

\section{Introduction}

This contribution is an extension of the previous development [1-3] of the Ateneo Innovation Center (AIC) in the development of a phototherapy system for the treatment of infants with jaundice. The system has been proven to be reliable and effective, with over 30 patients treated $[2,3]$ during clinical investigation. Our previous efforts described that this unit has been developed at far less cost and may achieve similar results like the commercially available units.

Neonatal jaundice (NNJ) occurs in $25 \%$ to $60 \%$ of full-term newborns and $80 \%$ of preterm newborns in the first two weeks of life, as studied by Cecelia Henny-Harry and her research partner [4]. It is a benign transient physiological event in the majority of newborns but can cause irreversible brain damage and kernicterus in some infants if the serum bilirubin levels are high. Various mechanisms involved in producing this 'physiological' increase in total serum bilirubin include increased production of bilirubin due to lysis of red blood cells, decreased ability of liver cells to clear bilirubin and increased enterohepatic circulation. Any condition

\footnotetext{
*Corresponding Author: Paul Cabacungan, pcabacungan@ateneo.edu
}

that further increases bilirubin production or alters the transport or metabolism of bilirubin increases the severity of physiological jaundice [5].

The main indicator of NNJ is the presence of high levels of bilirubin. Among the effects of high levels of bilirubin caused by rapid $\mathrm{RBC}$ metabolism is the increased levels of carbon monoxide in the body, which sometimes causes neurologic dysfunction [6]. Adults may also contract jaundice by having elevated serum bilirubin levels. Jaundice in adults is often an indicator of underlying condition, such as hepatitis or liver disease, and injury [7]. The recurrence of jaundice or its persistence can have astounding and long-standing effects on the health and overall welfare of the patient.

Some factors that increase the likelihood of contracting jaundice are genetics, hospital setting, and social-economic status. In addition, persons of African, Mediterranean, Middle Eastern, or Southeast Asian descent are more likely to contract jaundice [8]. With this in mind, we can infer that Filipinos belonging to lowerincome households will be more susceptible to this disease, and so 
are communities with little to no access to sufficient medical services.

Phototherapy is the most frequently used treatment when serum bilirubin levels exceed physiological limits. To initiate phototherapy without delay is the most important intervention for infants with severe hyperbilirubinemia. Phototherapy uses blue light to convert bilirubin into water-soluble photo-products that can bypass the hepatic conjugating system and be excreted without further metabolism. The clinical response to phototherapy depends on the efficacy of the phototherapy device, as well as the infant's rates of bilirubin production and elimination [9]. Phototherapy, despite being the recommended standard treatment for hyperbilirubinemia in newborns, may not be accessible to many people most especially in developing countries. This is due to its high procurement and maintenance cost ranging from PHP 150,000 to PHP 350,000 (USD 3,100 to USD 7,000) [10]. In the Philippines, for rural clinics and maternity hospitals to be accredited by Philippine Health Insurance Corporation (PhilHealth), a tax-exempt Government Corporation attached to the Department of Health that administer the National Health Insurance Program [11], they must possess an operating phototherapy system for jaundiced infants.

For more efficient operations, clinics and hospitals need a detailed digital record of consultation or confinement such as demographic data of the patient, identity of attending medical staff, medical and laboratory procedures and results, among others. In the case of NNJ, rural hospitals will need chemical reagents and a hematology analyzer to administer actual bilirubin counting of blood samples. Access to comprehensive patient digital records and to needed reagents and medical devices is usually problematic, especially in remote areas. When an infant is suspected to be suffering from $\mathrm{NNJ}$, the doctors resort to physical inspection to assess the existence or degree of jaundice.

In 2016, Ateneo Innovation Center together with its partner pediatricians conducted a two-year pilot study and clinical investigation to evaluate the capability of the LPLS in lowering bilirubin levels among healthy term infants diagnosed with indirect hyperbilirubinemia admitted at the nursery, OB ward, and pediatric ward of a tertiary hospital in Metro Manila. [2,3]. Figure 1 shows the LPLS administered by a pediatrician during the clinical investigation, after passing through all the necessary protocols required by the ethics committee.
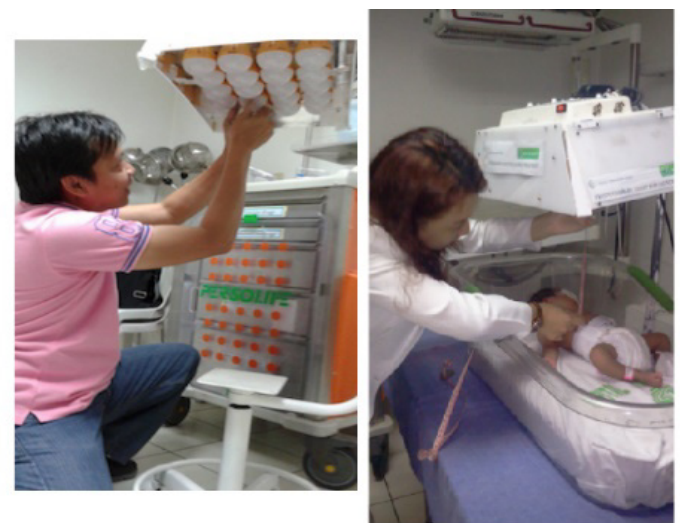

Figure 1: Infant diagnosed with indirect hyperbilirubinemia underwent phototherapy using the LPLS prototype in a tertiary hospital in Metro Manila, Philippines
The clinical investigation revealed that $16 \%$ of the total number of patients completed treatment after 24 hours of phototherapy under the LPLS and were classified to be in the low risk zone while $36 \%$ completed treatment after 48 hours. The total bilirubin significantly decreased by $16.5 \%(p=0.0001)$ from baseline bilirubin levels after 24 hours. There was a significant $29.9 \%$ mean percentage of reduction in bilirubin level after 48 hours. The proportion of subjects in the high-risk zone during baseline to 24 th hour went down significantly from $80 \%$ to $28 \%$ $(\mathrm{p}=0.0003$ ). In the same manner, comparing baseline to 48 th hour, the percentage of subjects in the high-risk zone went down from $80 \%$ to $9.5 \%(p=0.0001)$. Figure 2 shows the graphical representation of the results.

After the clinical investigation, there were no reported occurrences of rebound hyperbilirubinemia. No patient experienced any complication while on treatment. This makes the system safe and effective in lowering total serum bilirubin among healthy term infants with indirect hyperbilirubinemia [3].

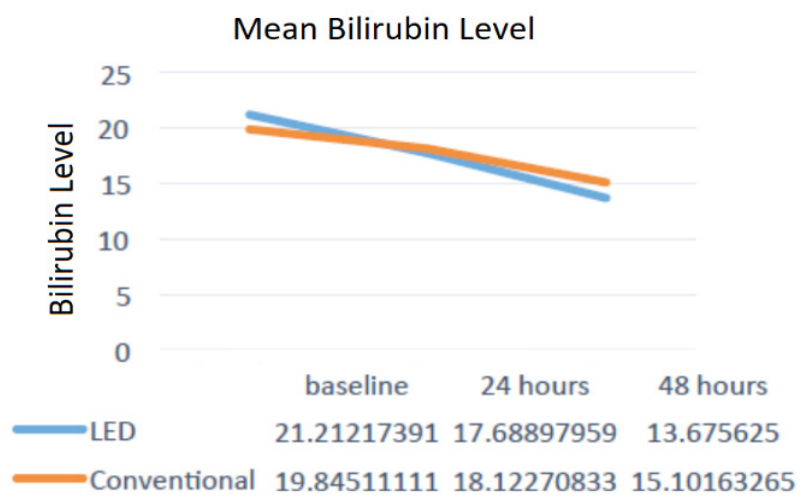

Figure 2: Mean TSB levels in both groups at different period [3]

Ateneo Innovation Center developed further the Low-cost Phototherapy Light System (LPLS), shown in Figure 3, with the integration of sensors making it an Improved Low-cost Phototherapy Light System (ILPLS) as seen in Figure 4. ILPLS was designed to have the same functions, to provide the same treatment, and to keep its low cost, as the original prototype.

The main components of the ILPLS were the eight pieces of 7-watt LED blue spotlight, which were more robust and had the same cost as the 3-watt bulbs of the LPLS. The wavelength was within the 400 - 500 nanometers (peak at $460 \mathrm{~nm}$ ) which are specifically used for administering phototherapy [12]. The measured irradiance was three times stronger than that of the 3watt bulb and its unit exceeded the total irradiance of the previous unit. The bulbs were positioned alongside each other at 13 centimeters apart (from center to center). This engineering design made the light beams overlap by $43 \%$ resulting in a more intensified lighting. There is an $11 \%$ intersection of light beams coming from the bulbs positioned across each other, which are 22 centimeters apart. The 22-centimeter distance was an engineering trade-off to give way to the fans that provide fail-safe mechanisms. During the 120-hour operation, the ILPLS bulbs performed in an acceptable temperature range of 35.8 to $39.0^{\circ} \mathrm{C}$, with ambient average temperature of 32.5 degree Celsius. No issues were encountered at all. An additional 10-watt computer fan was also installed. 


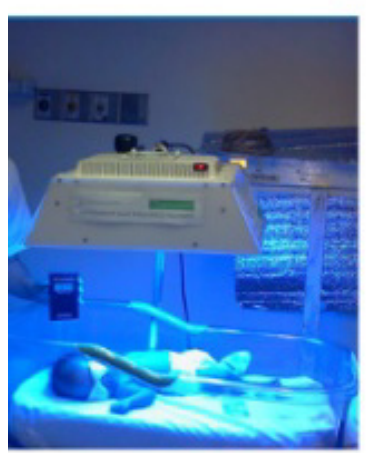

Figure 3: Low-cost Phototherapy Light System (LPLS)

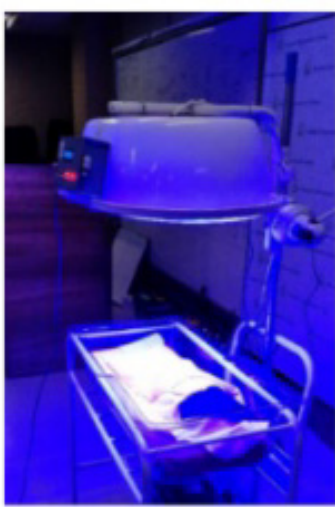

Figure 4: Improved Low-cost Phototherapy Light System (ILPLS)

The sensor and monitoring system were also introduced and successfully integrated into the ILPLS without affecting the unit's optimal functioning. The camera system is intended to provide digital records of the patient's ID, time stamp, unit tag and audio tags by medical personnel. The smart camera was able to transmit images and sounds within the 10 -meter radius in a laboratory setting, despite the presence of walls, demonstrating that data collection at nearby stations is possible. The location of the phototherapy unit is crucial to readily interface with nursing stations and hospital medical records. This approach is scalable and useful to situations where multiple phototherapy units are operated at the same time. It can easily record the data of the ongoing phototherapy and supplement the nurses' records. Four light dependent resistors (LDRs) were installed to ensure emission of appropriate light intensity. The two-in-one sensor was also able to measure temperature and humidity inside the light box. Alarms would signal readings beyond threshold, adding safety features to the system.

Ateneo Innovation Center acknowledges the long-standing problem of the people in remote and off grid communities who have little or no access to this device. Through the efforts of this organization, professors, students, and different organizations continue to collaborate and work together in developing innovative designs and ideas that provide solutions with impact on the betterment of society. This paper presents another Ateneo Innovation Center's development that aims to address a need in remote settings - the Smart Low-cost Phototherapy Light System (SLPLS) with non-invasive jaundice monitoring for neonatal jaundice.
Shown in this paper is how we are developing new sensor and monitoring systems that enhance the performance of our low-cost phototherapy light systems and facilitate data collection and sharing with patients' families and the stakeholders. Having a digital record to determine the progress and success of treatment, doctors can monitor and assess the progress of the treatment in a more scientific way. We are developing yellow jaundice sensors that can monitor the treatment of the patient and can provide a possible basis for detection. We explore different color sensor experiments, using accessible devices and open source applications that can be integrated into our latest prototype. We also demonstrate optical techniques for color measurement that can be a tool for obtaining a digital record of the degree of jaundice. Lastly, we deal with an important aspect of the development - solar powering of the phototherapy system for $24 / 7$ performance in case of power outage or blackouts and remote deployment. To further prove that this system is effective enough to be used as an alternative than that of a commercial unit, we have yet to conduct further tests with actual human subjects that will be supervised by medical personnel.

\section{System Overview}

This paper describes the most recent Smart LPLS prototype with further improvements to its electronic sensor and monitoring system. We also present non-invasive methods that will help determine the intensity of yellowness in infants as a way of detecting NNJ. The Smart LPLS is composed of eight 7-Watt LED bulbs at the top and the additional ten 10-watt LED tube light pieces at the bottom, as seen in Figure 5. This makes the system more robust compared to the 3-watt and 7-watt LED bulbs used in previous prototypes presented earlier. These additional T5 LED tube lights are $90 \%$ more efficient than traditional light bulbs, $60 \%$ compact than fluorescent bulbs, and have a lifespan of 30,000 hours

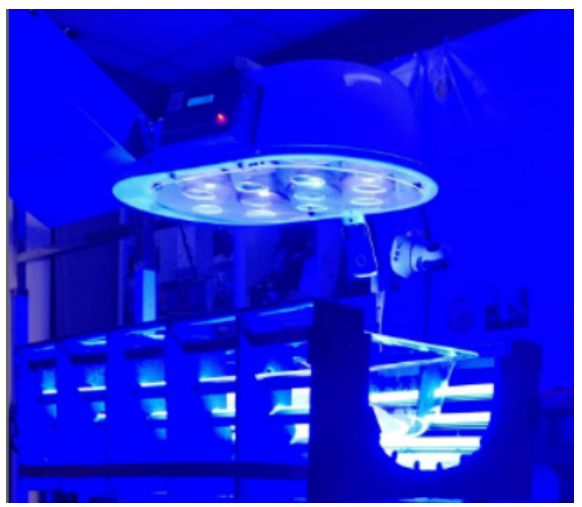

Figure 5: New Smart Low-cost Phototherapy Light System

To easily monitor the system's performance, an automated system that stores data about the device's light intensity and bulb temperature and records ambient humidity was installed. An alarm system that activates the warning lights if sensor readings are in critical level and if the bulbs need to be replaced are built into the system. A time setting feature is also included to manually set the time of operation and automatically turn-off the device. The system diagram for the smart sensor and monitoring system can be seen in Figure 6 . 


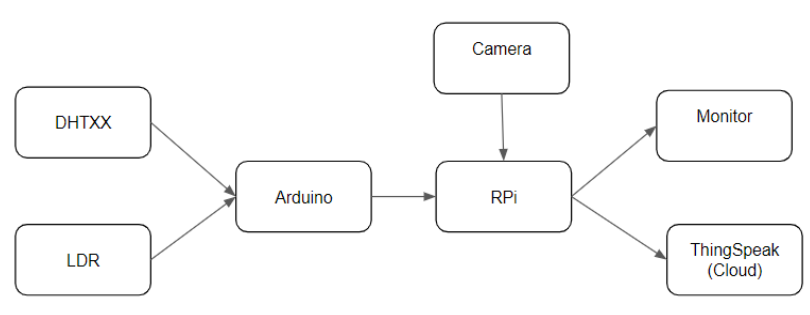

Figure 6: System diagram for the smart sensor and monitoring system

The DHT22 sensor, which can measure both humidity and temperature, is placed inside the system. Meanwhile, the photoresistors or the light dependent resistors (LDRs) are placed near the phototherapy light to monitor the light intensity. Both are connected to the Arduino which will send data to the Raspberry Pi later. While the Arduino is feeding sensor data to the Raspberry $\mathrm{Pi}$, a camera may also be connected to it. The Processing IDE will process the data and display everything in one window through the monitor where people can see if everything is as expected. Measured data like temperature, humidity, light intensity, and images are sent to ThingSpeak IoT platform, which can be used to send data to the cloud from any Internet-enabled device. Users can configure actions and alerts based on real-time data and unlock the value of data through visual tools.

The non-invasive jaundice treatment monitoring feature of the Smart LPLS aims to provide an alternative platform for physicians to conduct a non-invasive method to check how severe the jaundice of an infant is while on treatment. Blood analysis is currently used to determine bilirubin level in the blood. This process is invasive, and the results may take some time. Physical inspection may also be done but the accuracy of results depends heavily on the skill of the medical practitioner performing the inspection. In our experience, provincial hospitals often lack the necessary reagents to carry out these tests, necessitating an alternate measurement. Developing a tool that will determine the intensity of yellowish color in infants can give immediate and accurate detection of NNJ in a non-invasive way. Also, commercial phototherapy systems and bilirubinometers are expensive and rural hospitals might not have the financial resources to afford these. The sensor will be helpful in gauging and monitoring if the phototherapy treatment is sufficient and successful in treating the jaundice in infants. If phototherapy was not enough, blood transfusion might be needed. The system may store collection of readings and activate a warning light indicator if sensor readings that are related to bilirubin level go beyond the set ceiling, indicating that the condition cannot be managed through phototherapy light and a more aggressive treatment approach such as blood transfusion is needed. The same automation system can also activate a different warning light if sensor readings go below a set floor, indicating the full treatment of NNJ. This means that the infant can be removed from phototherapy light exposure.

In Table 1, we compared the prototypes with a commercially available unit. What sets the Smart LPLS apart from the previous prototypes are the top and bottom irradiation. This results in a $360^{\circ}$ light exposure, without the need to tilt the baby every two hours. Moreover, the irradiance of top-mounted lights is three times stronger than the minimum required irradiance of 30 $\mu \mathrm{W} / \mathrm{cm}^{2} / \mathrm{nm}$ for NNJ treatment. In addition, the bottom-mounted lights provide an irradiance of $34 \mu \mathrm{W} / \mathrm{cm}^{2} / \mathrm{nm}$, leading to an expectation for faster treatment. Despite the installation of more lights, the Smart LPLS has two fans with air vents to provide safety mechanisms.

The Smart LPLS for off-grid deployment with a total unit cost of PHP 38,000.00 or around USD 780.00 uses energy efficient LED bulbs and tubes having a full width half maximum wavelength range of $439.66 \mathrm{~nm}$ to $467.64 \mathrm{~nm}$ that is within the curing wavelength of $400-500 \mathrm{~nm}$, irradiance of $34 \mu \mathrm{W} / \mathrm{cm}^{2} / \mathrm{nm}$, with top \& bottom lights still within the minimum wavelength, additional air vents, sensors and monitoring system, and with integrated non-invasive yellow detection feature. We have listed our estimates of the baseline system costs, however, due to time constraints and the current setup of the research laboratory, the systematic optimization in relation with its cost discussing the reliability, longevity, and efficiency is of pending approval by medical authorities will be discussed in future publications.

Table 1: Comparison of Smart LPLS with its Predecessors and Commercial Unit

\begin{tabular}{|c|c|c|c|c|}
\hline Parameters & LPLS & ILPLS & Smart LPLS & $\begin{array}{l}\text { Commercial } \\
\text { Unit }\end{array}$ \\
\hline Light Bulb & $\begin{array}{l}\text { Twenty } \\
\text { 3watt } \\
\text { LED } \\
\text { bulbs }\end{array}$ & $\begin{array}{l}\text { Eight 7- } \\
\text { watt LED } \\
\text { spotlights }\end{array}$ & $\begin{array}{l}\text { Eight } 7 \text {-watt } \\
\text { LED and Ten } \\
10 \text {-watt LED } \\
\text { tubes }\end{array}$ & $\begin{array}{l}\text { Five 20-watt } \\
\text { Fluorescent }\end{array}$ \\
\hline Wavelength & $\begin{array}{l}462.1 \\
\mathrm{~nm} \text { to } \\
476.4 \\
\mathrm{~nm}\end{array}$ & $\begin{array}{l}437.8 \mathrm{~nm} \\
\text { to } 458.48 \\
\mathrm{~nm}\end{array}$ & $\begin{array}{l}437.8 \mathrm{~nm} \text { to } \\
458.48 \mathrm{~nm} \text {; and } \\
439.66 \mathrm{~nm} \text { to } \\
467.64 \mathrm{~nm}\end{array}$ & $\begin{array}{l}\text { ( } 5 \text { white and } \\
2 \text { blue) }\end{array}$ \\
\hline $\begin{array}{l}\text { Irradiance at } \\
30 \mathrm{~cm} \text {. average } \\
\text { distance }\end{array}$ & $\begin{array}{l}84 \\
\mu \mathrm{W} / \mathrm{cm}^{2} \\
/ \mathrm{nm}\end{array}$ & $\begin{array}{l}90 \\
\mu \mathrm{W} / \mathrm{cm}^{2} \\
/ \mathrm{nm}\end{array}$ & $\begin{array}{l}90 \text { and } 34 \\
\mu \mathrm{W} / \mathrm{cm}^{2} / \mathrm{nm}\end{array}$ & $\begin{array}{l}70 \mu \mathrm{W} / \mathrm{cm}^{2} \\
/ \mathrm{nm}\end{array}$ \\
\hline Total Power & 75 watts & 90 watts & 240 watts & 400 watts \\
\hline Ventilation & one fan & two fans & $\begin{array}{l}\text { two fans with air } \\
\text { vents }\end{array}$ & air-cooled \\
\hline $\begin{array}{l}\text { Sensor and } \\
\text { Monitoring } \\
\text { System }\end{array}$ & none & present & present & none \\
\hline $\begin{array}{l}\text { Integrated } \\
\text { Non-invasive } \\
\text { yellow } \\
\text { monitoring }\end{array}$ & none & none & present & none \\
\hline Unit Cost & Php20k & Php35k & Php38k & Php150k \\
\hline
\end{tabular}

Since 2015, the Ateneo Innovation Center has been studying and exploring the Near Cloud or Mobile Cloud, recognizing its powerful computing and caching capabilities in the context of disaster resilience, education, and healthcare. The concept of caching is powerful especially in a telecom infrastructure-less environment. This technology uses a combination of a plug computer, preloaded content, and key web applications on terabyte drives at fiber-like speeds. It addresses limited bandwidth and connectivity by providing valuable preloaded content and acting as a gateway to the Internet [13-15]. 


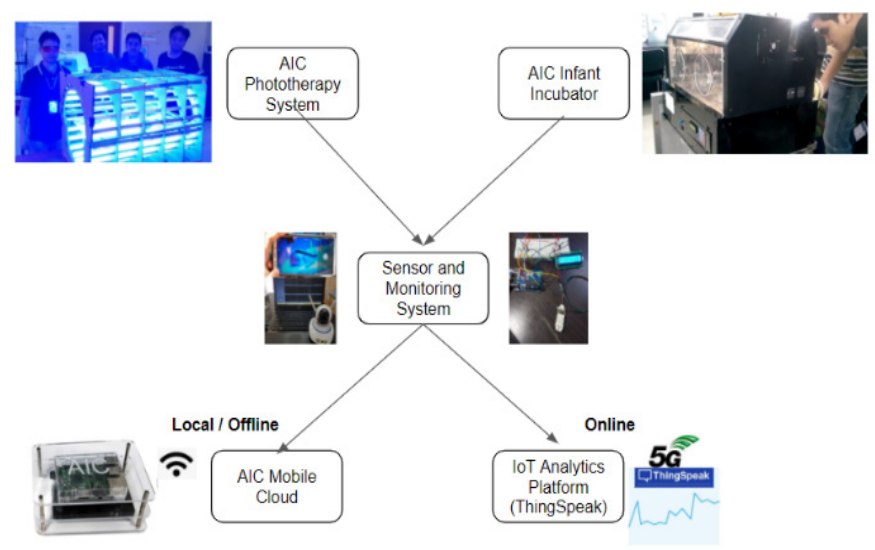

Figure 7: Smart LPLS as part of the envisioned Smart Nursing Station

We foresee this system to be part of a Smart Nursing Station, which diagram is in Figure 7, wherein a visual and auditory monitoring system is implemented to remotely oversee infants from the nurse station. The cameras will capture image and recordings of the infant that will serve as logs and be written in baby's medical records. It also sends alerts and data to the medical doctor or staff if there are any updates. Medical records and images of patients can be easily archived and retrieved if stored locally in the Near Cloud. Having the patient's data in the cloud also promotes interoperability and seamless transfer of data among the various stakeholders and speeds up healthcare delivery. This therefore closes the Medical Data Loop and promotes $5 \mathrm{G}$ and mobile cloud architectures in academic and business settings as well as for applications in sustainable and resilient communities [16].

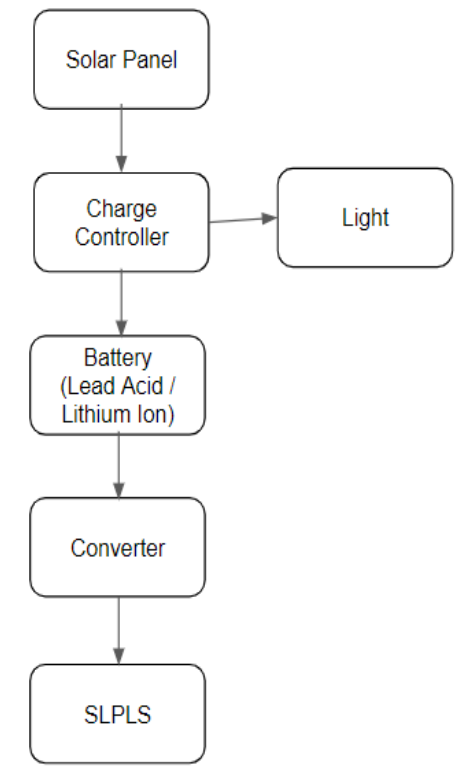

Figure 8: Off grid setup for Smart LPLS

In our previous study, we were able to run the system for 10 hours powered by two 50 -ampere-hour commercial lead acid car batteries that were pre-charged by two 50 -watt solar panels [1]. In this study, we continue to explore ways to make the system energy-efficient to be easily supported by solar powering. Ultimately, we hope to deploy the Smart LPLS in remote areas using the set-up shown in Figure 8.

\section{Review of Related Literature}

Total body phototherapy exposure (TBPE) is a new and effective technique to avoid the risks of invasive exchange blood transfusion (EBT) in neonates with jaundice. TBPE consists of top- and bottom-mounted lights to provide whole body phototherapy light exposure. Ogah Emeka Onwe and his research team in 2019 used an imported phototherapy light unit that has an average spectral irradiance of $34 \mu \mathrm{W} / \mathrm{cm}^{2} / \mathrm{nm}$ and 50 $\mu \mathrm{W} / \mathrm{cm}^{2} / \mathrm{nm}$ in the top and bottom lights, respectively, and a peak wavelength of $455-470 \mathrm{~nm}$. It was reported that out of 82 severely jaundiced cases, all TBPE cases were successfully discharged except for two mortalities due to related exchange blood transfusion complications [17].

In general, the same authors found that TBPE is an efficient and a good non-invasive alternative to EBT intervention [17]. It is also affordable for resource-constrained settings. But some limitations were encountered during the study. The frequent occurrences of unannounced power failures at the hospital facility might have adversely affected the overall treatment time. Also, the identification of qualified subjects for inclusion in the study was limited due to poor record keeping.

In determining jaundice condition in infants, the practice of visual assessment is quite subjective and needs a lot of experience from the medical practitioner to determine severity of jaundice condition, as stated in The Journal of Pediatrics by Arieh Riskin and his co-researchers [18]. Thus, medical practitioners can use devices which can measure the levels of jaundice in infants without blood analysis. Infants under treatment can still have increased bilirubin levels which is very dangerous when not recognized immediately. On the other hand, infants with decreased bilirubin levels can only be verified upon examining the blood. In the absence of total bilirubin serum (TBS) test and hematology analyzer, a database of infant's yellowish skin color across varied skin tones may be helpful in the visual assessment of the patients' condition.

Methods of digitally sensing color pigment from reflected light may offer a simple and objective way of determining the existence or severity of jaundice condition during visual inspection. Current procedure of detecting jaundice in infants, such as newborn screening (NBS) test, is done by physicians through visual inspection and blood analysis to measure the bilirubin level. The study of Hafizon Baharuddin and his group in 2010 presented an infrared sensor that detects bile pigments, but blood extraction is still needed to assess the bilirubin level [19].

A method for measuring jaundice using non-contact optical devices at the conjunctiva of human subjects was described by Nabarun Polley and his colleagues in 2015 [20]. The light from the source is transmitted through the six excitation fibers of the excitation arm and incident on the subject (conjunctiva). The diffused light is collected by the detection fiber and transmitted through the detection arm to the spectrograph. The spectral response corresponding to the conjunctiva is processed and generated in the laptop computer as shown in Figure 9 below. 


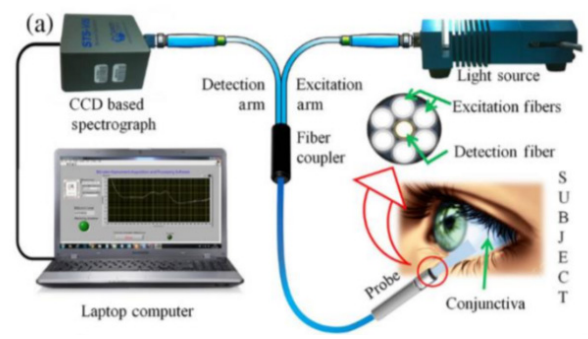

Figure 9: Use of non-contact optical devices at the conjunctiva of human subject

In the figure below, the comparative spectral response of a normal volunteer and a jaundice patient using the wavelength calibration of their setup has been established with a He-Ne laser $(632.8 \mathrm{~nm})$, fluorescent lamp, and emission/absorption of a number of dyes including aqueous bilirubin solution are shown. A distinct difference in the spectral appearance is visible; the contribution of yellow pigment deposited in the conjunctiva of the jaundice patients is higher compared to the normal volunteer. An absorption spectrum of aqueous bilirubin solution is also included in Figure 10 as reference.

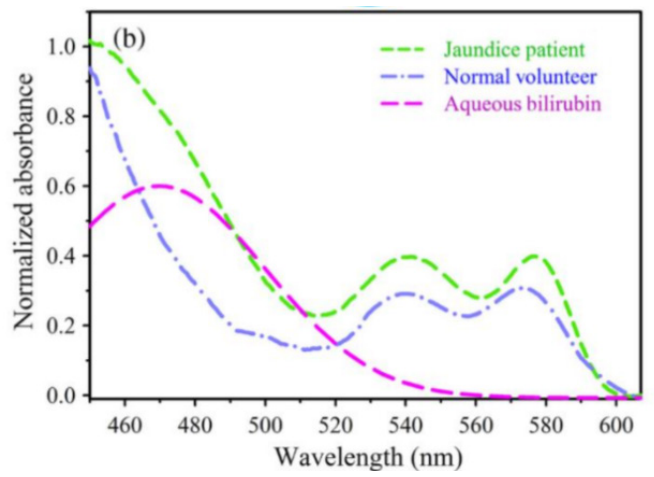

Figure 10: The comparative spectral response of conjunctiva of a normal volunteer and jaundice patient

They have also developed a non-invasive and practical device for measurement of bilirubin levels. While serum bilirubin measurements are still required for precise diagnosis, the proposed device shows the potential that reduce frequent blood sampling. This procedure may be difficult to use knowing that newborns sleep most of the time and their eyes are small and sensitive.

\section{Results and Discussion}

This is a design and engineering study with no infants or humans involved, with no cross-reference with real jaundice patients and no photos during treatment and corresponding bilirubin measurements were taken due to ethics concerns and time constraints. This study was partially funded by Ateneo de Manila University, where the authors are affiliated with, so there is no conflict of interest to be declared.

\subsection{Increased Surface Exposure}

The main component of the phototherapy treatment is the blue light which is provided by the blue LEDs. Using LEDs also reduces the risks of complications such as progressive skin damage brought about by conventional ultraviolet light used by standard phototherapy light treatments. To ensure the efficiency and effectiveness of the device, light intensity, the distance from the subject of phototherapy, the area of exposure, the duration of the treatment and the amount of bilirubin present in the body of the subject must be taken into consideration.

The blue light source in the Smart LPLS is coming from the top-mounted spotlights and bottom-mounted tube-type LEDs. The Smart LPLS has a wavelength that peaks at $253.99 \mathrm{~nm}$ and has a full width at half maximum (FWHM) range of $439.66 \mathrm{~nm}$ to $467.64 \mathrm{~nm}$. Its irradiance was measured from an oval grid with a length of $60 \mathrm{~cm}$ and width of $40 \mathrm{~cm}$ from 35 different data points. The average distance of the measuring device (RS Pro) from the phototherapy light mimics the distance of the infant patient from the phototherapy light which is about $30 \mathrm{~cm}$. The measured irradiance is $34 \mu \mathrm{w} / \mathrm{cm}^{2} / \mathrm{nm}$, which is also above the minimum required irradiance of $30 \mu \mathrm{w} / \mathrm{cm}^{2} / \mathrm{nm}$.

The additional set of phototherapy lights installed under the transparent bassinet also acts as the frame and stand of the whole system. The existence of bottom-mounted lights provides additional irradiation that may shorten treatment time.

\subsection{Monitoring of Neonatal Jaundice Patient}

One of the significant features of the Smart LPLS is the color sensor to monitor the treatment progress of the infant with jaundice based on the intensity of skin yellowness.

We are convinced in the importance of having a patient monitoring process when the phototherapy light system is deployed in remote areas without reagents and hematology analyzer. The cost of transcutaneous bilirubinometer (TcB) is similarly prohibitive making it difficult to find in remote areas.

This paper presents the use of inexpensive electronic sensors and devices that can detect even small color changes in the infant's skin by image processing. A low-cost method of skin tone simulation that is digitally generated and classified to detect change in yellow pigments can also address the restrictive financial resources in the rural areas. The sample size is limited only to the number of images we have conducted in our initial tests. Though the tests exhibit promising results, the data gathered are patient specific since we covered only three colors of skin.

\subsubsection{Profiling the Sensors}

For the non-invasive jaundice monitoring feature of the Smart LPLS, we utilized HD web camera and Raspberry Pi, Arduino, and color sensors, and RGB phone app to monitor patients noninvasively. We implemented the yellow detection feature by quantifying the reading of different sensors based on their profile.

\subsubsection{Creation of Yellow Swatches}

Due to time and ethical constraints, especially during this COVID-19 pandemic, yellow level detection tests using different sensors were done using printed yellow swatches, not using human subjects. We created a standard swatch of yellow that varies from $0 \%$ to $100 \%$ with increments of $10 \%$. We also tested the swatches with plastic film to compare it with different opacities. We are envisioning that doctors can use this plastic film or transparency swatches by putting it on a non-jaundice skin (with similar tone as the infant's skin) and comparing it with the 
level of yellowing in a jaundiced infant's skin. The jaundiced infant's condition can be initially verified through blood test and correlate with the increments of yellowness represented in the swatches. The image or data can then be evaluated by the supervising doctor remotely or check the history of treatment of the baby.

RGB color picker was used to make yellow swatches that will be detected by the sensor and the camera. It is based on the color theory that all visible colors can be made using the additive primary colors of red, green, and blue. Hue $(\mathrm{H})$, saturation $(\mathrm{S})$, and value $(\mathrm{V})$ are the main color properties that allow us to distinguish between different colors.

Hue is described by the dominant wavelength and is the first item we refer to when adding in the three components of a color and it refers to a color having full saturation. value refers to the lightness or darkness of a color. Value on the other hand indicates the quantity of light reflected. Saturation lastly defines the brilliance and intensity of a color [21]. In color theory, a tint is a mixture of a color with white, which reduces darkness, while a shade is a mixture with black, which increases darkness. Both processes affect the resulting color mixture's relative lightness [22].

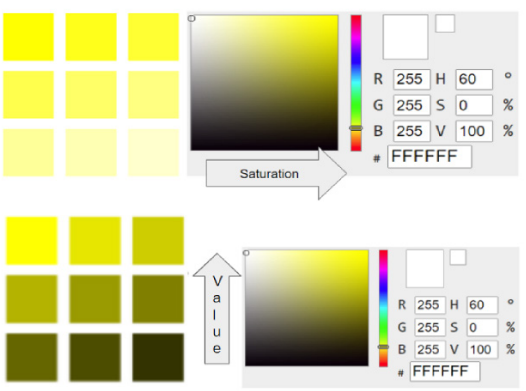

Figure 11: Different levels of Saturation (S) and Value (V) to obtain yellow swatches

Changing the $\mathrm{S}$ while maintaining $\mathrm{H}$ and $\mathrm{V}$ in constant values will give us different saturation levels of yellow. Also, shades of yellow in RGB have R and $G$ values at 255. As the transparency decreases, $B$ value increases from 0 to 255 . At $100 \%$ transparency, $\mathrm{B}$ is at its maximum value at 255 , while $0 \%$ transparency is at minimum value at 0 .

\subsubsection{TCS3200/TCS230 RGB Color Sensor}

In building the intelligent system, codes were developed to be used for the microcontrollers, sensors, and actuators. Calibration and testing of the system were also conducted to ensure the efficiency of the system. To jumpstart the tests using a detector to gauge the yellowness level of the skin, we used the TCS3200 / TCS230 RGB color sensor that has an array of photodiodes with 4 different filters and is mostly used for color recognition projects and can detect a wide variety of colors based on wavelength [23]. By selectively choosing the photodiode filter's readings, we were able to detect the intensity of the yellow and other different colors. This sensor has a current-to-frequency converter which converts its readings into a square wave with frequency that is proportional to the light intensity of the chosen color [23].

www.astesj.com

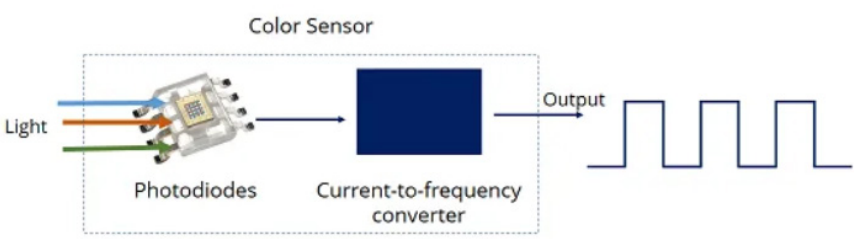

Figure 12: TCS3200 / TCS230 color sensor [23]

The sensor was connected to the Arduino and was able to receive serial data RGB values ranging from $0-255$. This sensor can accurately reproduce colors which were monitored by getting output from the Arduino. Processing3 IDE was used to verify by sight the similarity of the actual color and sensed color by taking in the serial data from the sensors, and visualize the color seen by the device by using the RGB value attained, and displaying it on a computer screen.

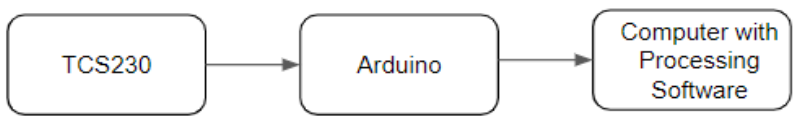

Figure 13: Block diagram for basic TCS3200 / TCS230 sensor test

Initially, we used a clear filter and set the control pins S2 and S3 to HIGH and LOW, respectively. We calibrated and enclosed the color sensor in a box to prevent ambient light from contaminating the sensor and concentrate the light reflected to the sensor.
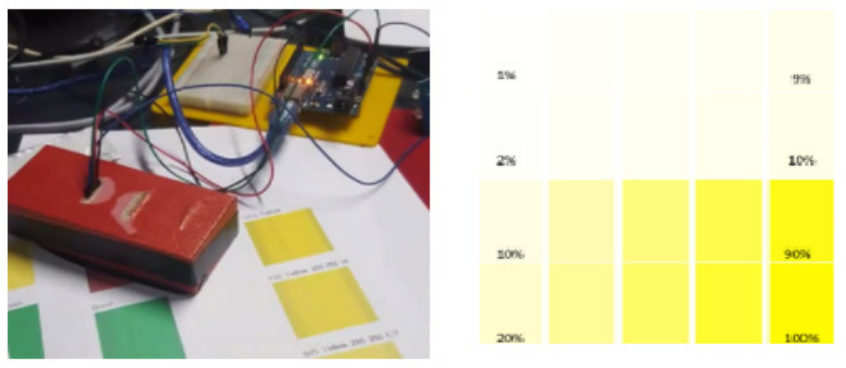

Figure 14: Enclosed TCS3200 / TCS230 and color swatches

Running the serial monitor will provide values that are dependent on the selected frequency-scaling and the condition of ambient light. The R, G, and B values differ due to the different sensitivity of each photodiode type, as seen in Figure 15. To represent the detected colors with the RGB Model with values from 0 to 255, we will use the map() function to map or convert the readings to values from 0 to 255 .

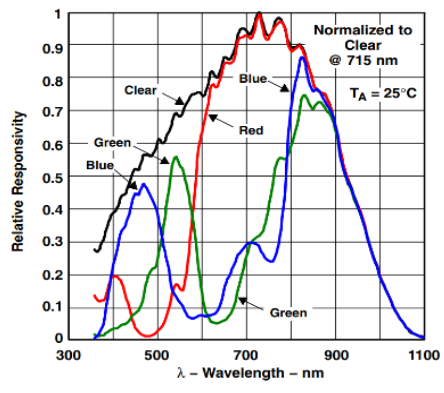

Figure 15: TCS3200 / TCS230 spectral responsivity diagram 
We profiled and checked the sensor to set baseline data. The graph in Figure 16 shows that the sensor can detect colors normalized to different values. The sensor is more responsive to more colors.

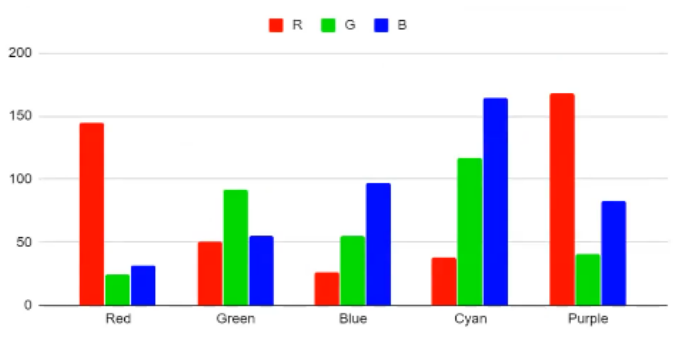

Figure 16: TCS3200 / TCS230 color profiling

We interpreted the theoretical RGB values for different shades of yellow as seen in Figure 17. Both R and G values are at 255 while $\mathrm{B}$ is at 255 if the color is at $0 \%$ opacity or $100 \%$ transparency. Meanwhile, B is at 0 when the yellow swatch is at $0 \%$ transparency. Once transparency increases, B increases as well, at a linear rate. Using this information, we compared the graph obtained by the blue values of the sensor, to the true-blue values of yellow swatches.

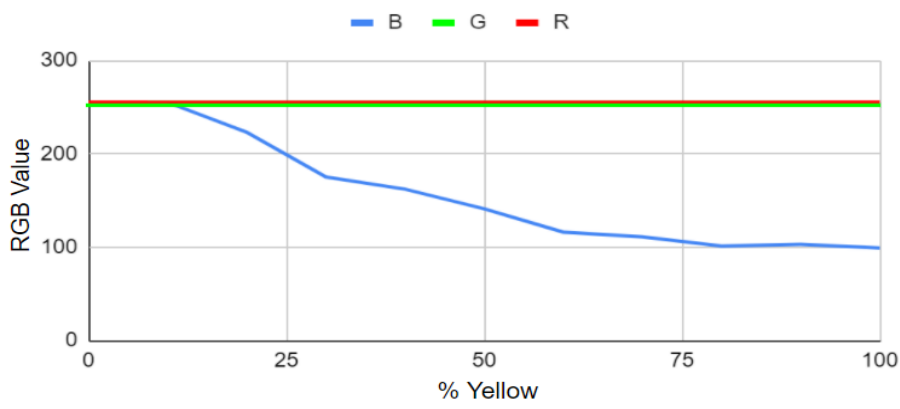

Figure 17: Theoretical and expected RGB values with different yellow values

As expected, a downward curve is shown in Figure 18 with $0 \%$ to $60 \%$ yellow of sensor values in line with the theoretical values, but it started to deviate from the theoretical value at around $60 \%$ yellow. We found that the sensor was able to accurately determine the $\mathrm{B}$ values of the swatches ranging from $40 \%$ to $100 \%$ transparency. The yellow pigment absorbs blue light and it allows red and green light to pass through or reflect from its surface.

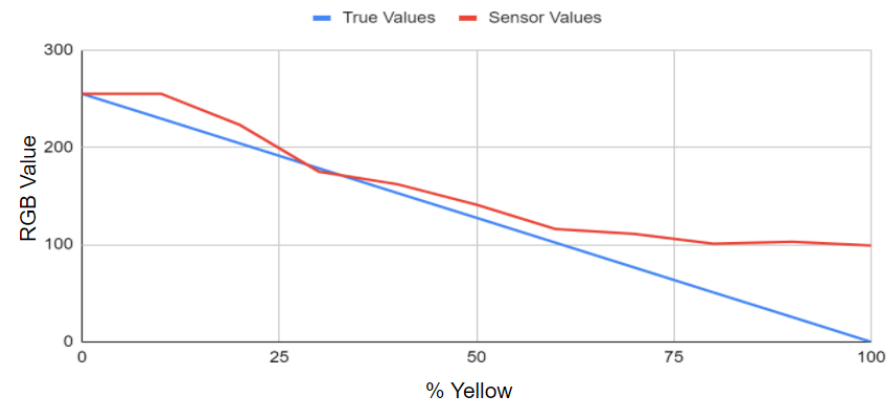

Figure 18: Theoretical vs sensor values of B values using without color filter

We also applied various color filters to the TCS3200 sensor and saw any changes or improvements in performance. In Figure www.astesj.com
19 , the experimental setup using the color filter of the sensor was shown. The built in LED of the sensor as the light source will shine light in the yellow swatches. Light will be bounced off the color filter that will be detected by the TCS3200 / TCS230 sensor which will generate a pulse width modulation data will proceed to the Arduino and be sent in the serial monitor.

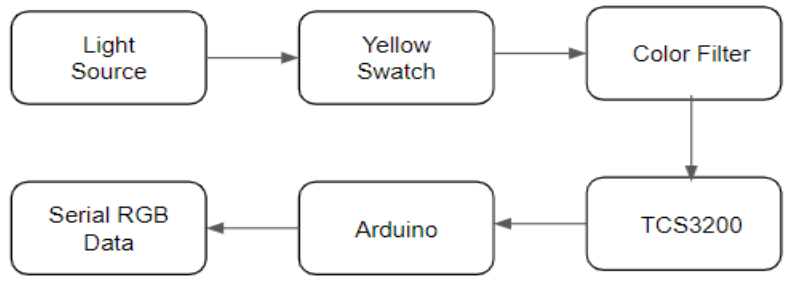

Figure 19: Experimental setup using TCS3200 / TCS230 sensor with filter

In Figure 20, a blue filter was placed in between the swatches and the sensor. For the blue level to be detected by the photodiode, we set the control pins S2 and S3 to LOW and HIGH, respectively. Green and red are not in constant 255 because of the filter and blue, as expected, the graph also shows a downward curve. It can be observed that blue dips in accuracy past $30 \%$ and none of the values follow the ideal relationship between blue values and yellow percentage.

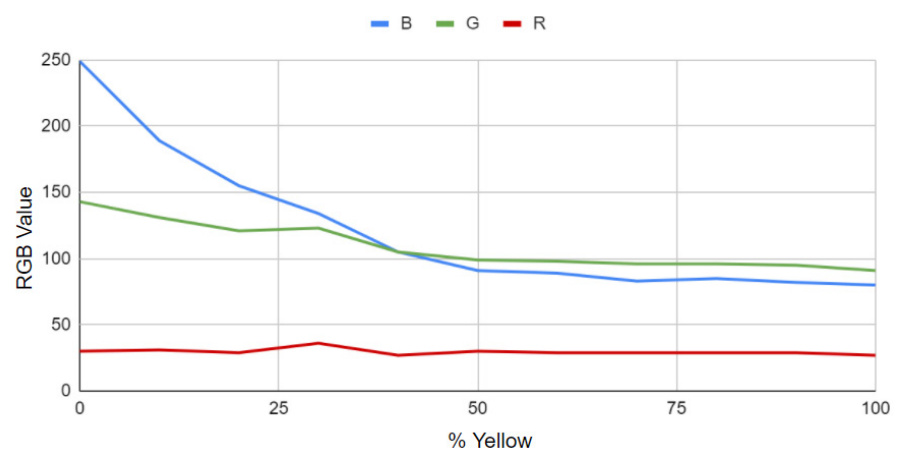

Figure 20: TCS3200 / TCS230 sensor using blue filter

None of the values are constant, but blue seems to be decreasing in a similar curve as it did with the other test. Looking at the results from the blue filter and no filter tests, blue values from the sensor seem close to the true values from $0 \%-60 \%$.

\subsubsection{ISL29125 RGB Color Sensor with IR Filter}

We also conducted tests and determined the performance of the ISL29125 sensor without filters and compared it to the true RGB values and each other. ISL29125 is a low power, high sensitivity, RGB color light sensor with an I2C interface and operates at 3.3V TTL and $56 \mathrm{uA}$ typical supply. Its state-of-theart photodiode array provides an accurate RGB spectral response and excellent light source to light source variation. It has integrated IR filter and compensation and outputs the actual light intensity with analog-to-digital converter and is designed to reject IR in light sources that allows the device to operate from well-lit to dark rooms. All the features of the device are controlled by the registers. The device is designed for operation under dark glass cover which significantly attenuates visible light and passes the infrared light without much attenuation. The device has an onchip passive optical filter designed to block or reject most of the 
incident infrared. This sensor is often used in ambient light color detection and / or correction [24].

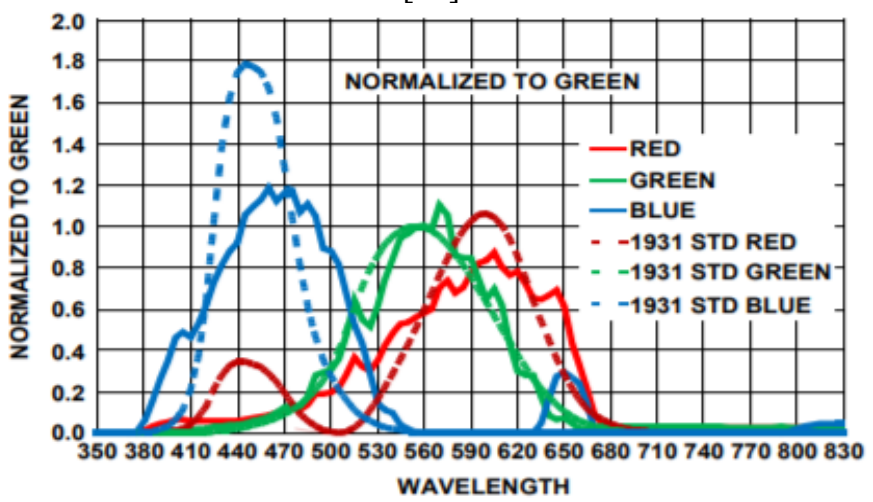

Figure 21: ISL29125 responsivity [24]

Housing for the sensor was created to keep out any ambient light from interfering with the received data. Using the ISL29125 with $\mathrm{I} 2 \mathrm{C}$ interface can be both an advantage and disadvantage as it operates at 3.3V TTL and a logic converter is needed if Arduino is used since the datasheet specifies $4 \mathrm{~V}$ as absolute max voltage while using less pins. An LED in series with a $510 \mathrm{Ohm}$ resistor was used because there is no built-in light for the sensor.

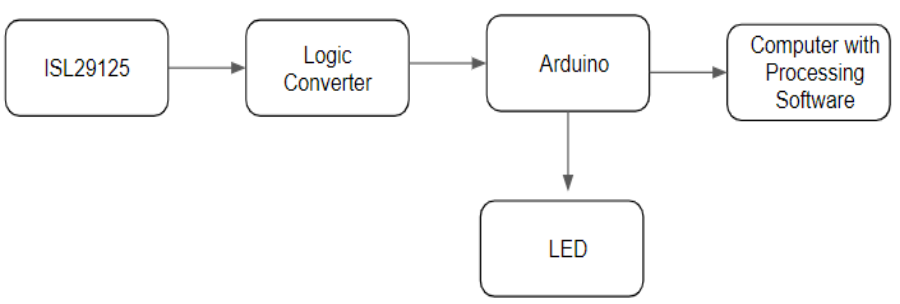

Figure 22: Block diagram for ISL29125 color sensor test

The sensor has three configurable settings. At CONFIG1, mode can be picked that determines what colors does the sensor samples, light intensity range from 375 lux to 10,000 lux depending on lighting condition, choose between 12 or 16 bit $\mathrm{ADC}$ precision depending on speed and precision, and sets the behavior of the interrupt pin if to be used as an input that triggers sensor sampling. CONFIG2, on the other hand, provides the option to set the level and range of active infrared compensation and lastly CONFIG3 that sends an interrupt to the Arduino once RGB values reach a certain threshold. In this test, we used the default configuration by enabling all the RGB, set the light intensity range to 10,000 lux, 16-bit ADC, high IR compensation on the lower range, and no interrupts.

We also profiled and checked the sensor to set baseline data. The graph in Figure 23 shows that the ISL29125 sensor can detect colors normalized to different values. The sensor has slower deviation to colors as compared to the TCS3200.

The ISL29125 was used in determining the RGB values in different yellow swatches and it shows in Figure 24 that Red is constant at 255. Green, on the other hand, showed a downward trend from 232 at $25 \%$ yellow to 184 at $100 \%$ yellow. Blue, as expected, followed a downward trend as well from 161 at $25 \%$ to 63 at $100 \%$ yellow.

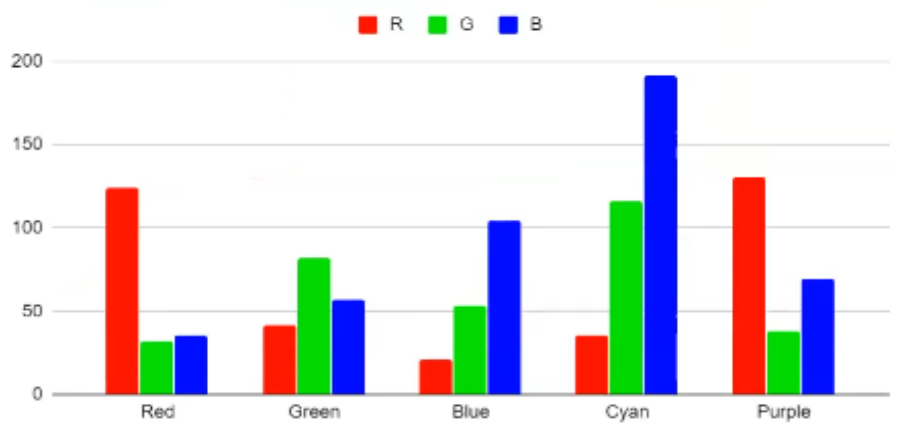

Figure 23: ISL29125 color profiling

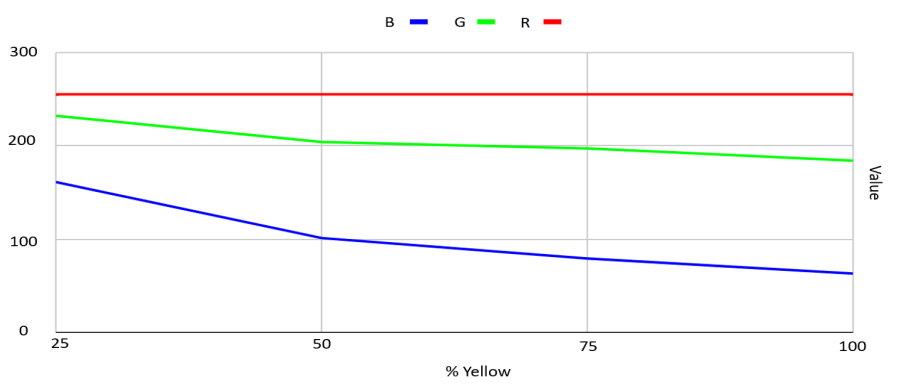

Figure 24: Test using ISL29125 sensor

Instead of using a white LED to illuminate the subject or surface, we used a red LED to test the reflectance of the surface for a specific color. In theory, red should reflect some, if not all, the red LED's light and sensor readings should be relatively higher compared to those that absorb instead of reflecting. This was seen in the data where the sensor read Red from colors where the red values were maxed out.

As seen in Tables 2 and 3, white, red, yellow, and purple swatches reflected red as expected. Values correspond to their measured RGB values and by looking at their full measured Red data of the swatches with red illuminant, white has the highest red value measured tied with yellow, then followed by purple, and lastly red. This order is also seen when measuring the colors using a white illuminant where White has the highest red value with red reading the lowest. Whether a white or red illuminant is used, the measured values are proportional in that their order from 'most red' to 'least red' is the same regardless of the illuminant.

Table 2: ISL29125 with red LED illuminant

\begin{tabular}{|c|c|}
\hline Swatch Color & Red Value \\
\hline White (\#FFFFFF) & 68 \\
\hline Red (\#FF0000) & 44 \\
\hline Yellow (\#FFFF00) & 66 \\
\hline Purple (\#FF00FF) & 58 \\
\hline Black (\#000000) & 0 \\
\hline Cyan (\#00FFFF) & 0 \\
\hline Green (\#00FF00) & 0 \\
\hline Blue (\#0000FF) & 0 \\
\hline
\end{tabular}


Table 3: ISL29125 with white LED illuminant

\begin{tabular}{|c|c|}
\hline Swatch Color & Red Value \\
\hline White (\#FFFFFF) & 255 \\
\hline Red (\#FF0000) & 124 \\
\hline Yellow (\#FFFF00) & 255 \\
\hline Purple(\#FF00FF) & 130 \\
\hline
\end{tabular}

The other colors had 0 color red reflected as detected by the sensor because by theory, they did not reflect red. This was done only with one red LED and compared to the white LED used in the earlier tests. Since the highest measured value is only 68 for red illuminant as to 255 of the white illuminants, the red LED might be too dim compared to the white illuminant. For further tests, it is recommended to install more LEDs for illumination since its dimness may not reflect the whole swatch or sample picture. It may be possible that the other colors do not reflect red but realistically they still could reflect a little red due to the color accuracy of the printers. Other colors may also be tried as well such as the suggested pulsing of Red and Green for Yellow. Proper calibration is also needed as the sensor's datasheet specifies very specific methods and processes to calibrate.

We also conducted an IR compensation test using the ISL29125 sensor which provides a programmable active IR compensation which allows fine tuning of residual infrared components from the output, which allows optimizing the measurement variation between differing IR-content light sources. There are two ranges set by B7:0 in the compensation register. In the library they are labeled as Offset and No Offset. B5:0 configures the specific value within the ranges. The preset values are HIGH, MED, LOW, but any 6-bit value can be set as the compensation. This combined with the offset gives 128 possible values for the active IR compensation. Figure 25 shows a typical system measure for both IR Comp Adjust and IR Comp Offset.

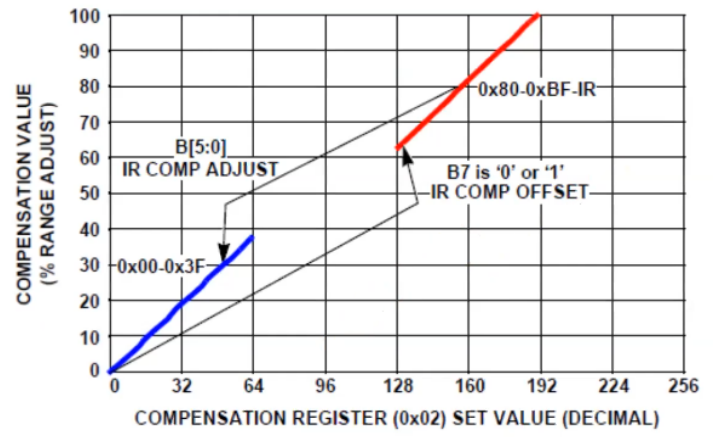

Figure 25: IR compensation set [24]

Using yellow swatch in this IR compensation test, Red and Blue have constant values as seen in Figure 26. When testing on colors, only the green color varied. With the highest IR compensation, the value of green is at its lowest. On the other hand, if there is no IR compensation set, green is at its highest.

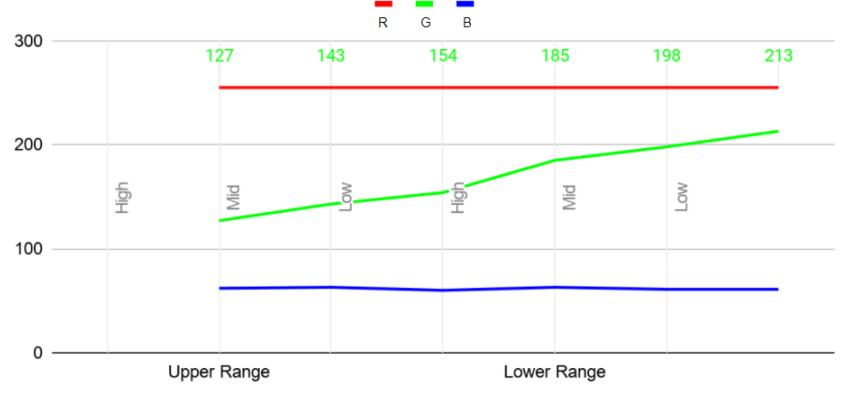

Figure 26: IR compensation using ISL29125 sensor

To test the difference on how the IR compensation works, the sensor was used on the same swatch while changing the IR modes between measurements. The measured values are seen in Figure 27. Since this printed color swatch was used, the most accurate value is the one with no offset / IR compensation.

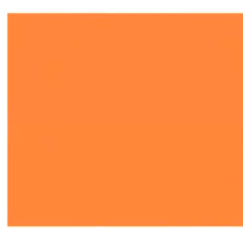

Offset - High

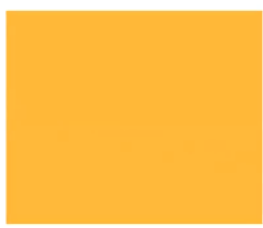

No Offset - High

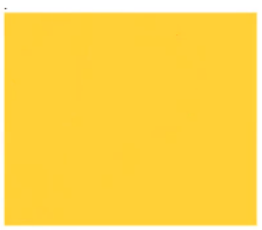

No Offset - Low
Figure 27: IR compensation using ISL29125 sensor

In these series of tests using sensors, both TCS3200 and ISL29125 perform similarly and the deciding factor would be whether configuring the sensor is needed. The power draw of the ISL29125 may be considered as it can be set to standby / power down mode to consume much less power. Another consideration is the footprint of the two sensors, the TCS230 is bigger and has 4 LEDs mounted. From the documentation, the ISL29125 is designed for operation under dark glass cover which could be an option and the purpose of the IR configuration.

\subsubsection{Web Camera}

For yellow color detection using a camera, we obtained image samples for different yellow levels using enclosed camera set-up and gathered its RGB values and used the weighted sum to quantify color level. Using off-the-shelf web camera is a low-cost platform in detecting the yellow levels of NNJ among infants.

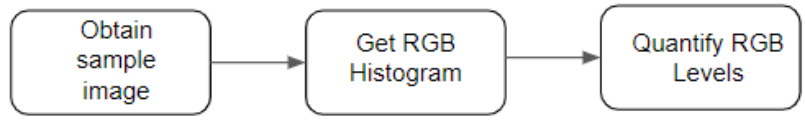

Figure 28: Flowchart for yellow color detection using camera

For this test, we used A4Tech PKS 730G (480p) Web Camera with $16 \mathrm{MP}$ camera sensor which supports up to $480 \mathrm{p}$ and with built-in LED. The camera was enclosed in a cup to prevent stray light from being detected by the camera. The camera could be connected to a laptop or low-cost Raspberry Pi computer via USB. The captured photo was then loaded to Processing software to 
obtain the histogram of the image, generate a CSV file and quantify the $\mathrm{R}, \mathrm{G}$ and $\mathrm{B}$ values using weighted average technique.

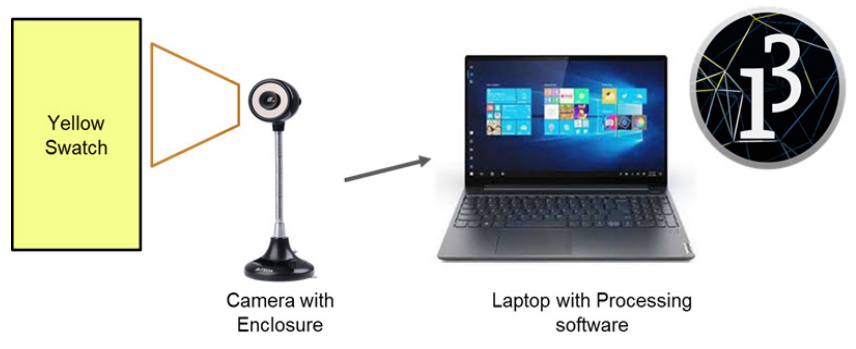

Figure 29: Test setup for yellow color detection using camera

Sample images from different levels of yellow opacity $(0 \%$ most transparent; $100 \%$ most opaque) were obtained using the camera and the RGB values, using weighted average of the RGB histogram, were recorded as shown in Figure 30.

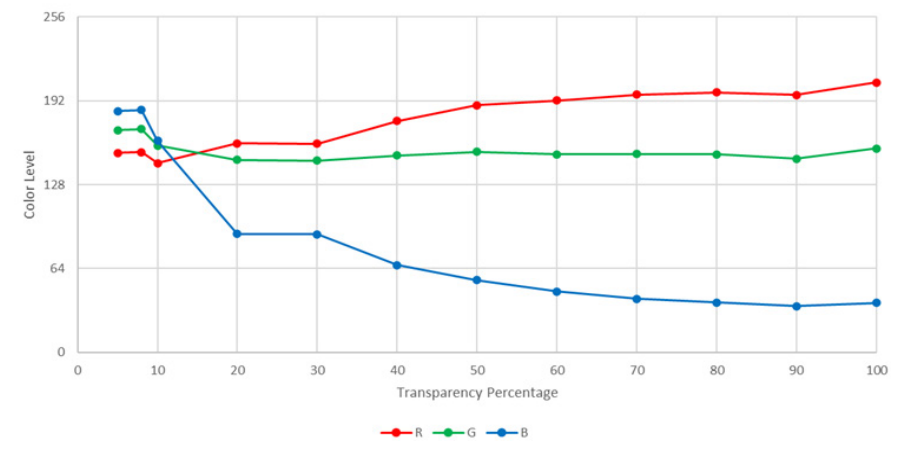

Figure 30: Different yellow levels for A4Tech PKS 730G

Theoretically, the red and green levels must remain constant and the blue level decreases as the yellow opacity level increases. From Figure 30, it could be observed that the red and green values started to stabilize at $50 \%$ yellow while values of blue followed a downward curve.

We expect fewer blue values as the blue color is absorbed by yellow, like how the phototherapy treatment works. To simulate the condition of a patient with NNJ, pictures of children with white, brown, and black skin tones were taken. The image used for the white skin tone swatch was from pexels.com, a free stock photo sharing website [25] while for the brown and black skin tone photo was from the photo collection of the author.

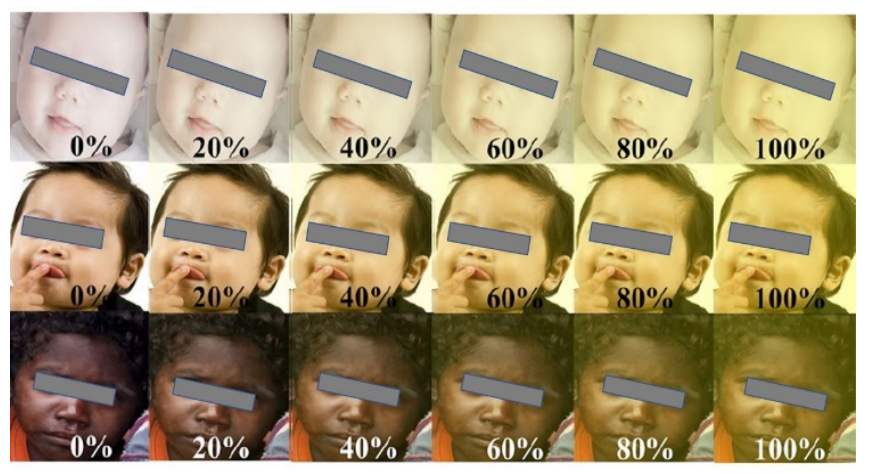

www.astesj.com

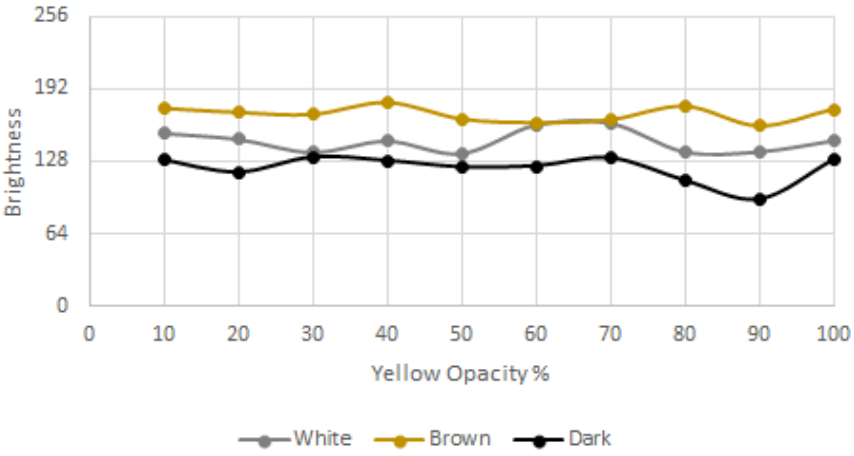

Figure 32: Red level for different baby skin types with yellow overlay

The yellow level corresponded to the opacity level of the yellow color. The swatches were built using Adobe Photoshop and were printed on a transparency sheet. The forehead area was chosen because face pictures were more available, and the said area had a strong correlation with serum bilirubin, as stated by Gwendoline Chimhini and her research collaborators [26].

In Figure 32, the brown skin type had a higher red level detected by the camera due to the brown color having red as the dominant color (brown $\mathrm{RGB}=150,75,0$ ). This test did not consider the reddening of the baby, particularly the white baby, due to blood or other sickness.

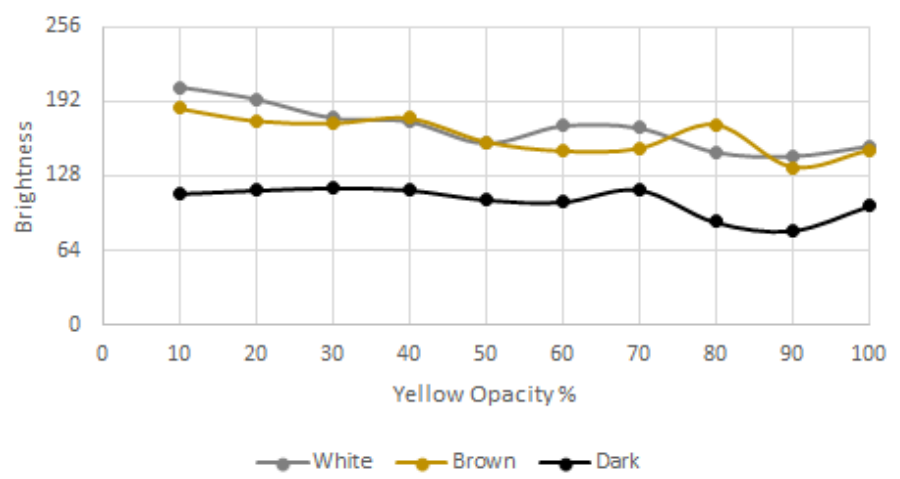

Figure 33: Blue level for different baby skin types with yellow overlay

In Figure 33, the blue levels detected for white and brown babies with yellow transparency overlaid were increasing as the yellowing of the baby decreased. This was due to the yellow color absorbing the blue. The decrease in yellow increased reflection of the blue level. However, for the black baby, no significant change in blue level was observed.

The improved electronic sensor and monitoring system complements the ability of doctors in observing and managing the condition of infants with $\mathrm{NNJ}$ and assists the doctors and patients in having a digital copy of medical records. In the future, we will explore and record various skin tones and nail colors of Filipino infants in photos as reference. The detection of the intensity of yellowness will be tested by placing different shades of yellow pigment over varied skin tones or nail colors.

\subsubsection{Smartphone RGB Color Application}

We also explored the use of Color Meter app, a simple color meter free mobile application that allows users to pick live colors and displays RGB color on the screen and the hexadecimal 
(HTML) color code that is used in graphics and web applications that can detect changes in RGB values. We used a Samsung S9+ smartphone with $f / 1.5$ and $f / 2.4$ (Dual Aperture) 12-megapixel camera and to explore the RGB values at zero and $100 \%$ yellowness.

The changes in RGB values are detected upon overlay of different levels of yellowness ( $0 \%$ to $100 \%$ yellow) on varied skin tones represented by white-, brown- and dark-skinned photos of infants, as seen in Figure 34. The graph shows the different amount of reflectance of RGB colors upon overlay of zero percent yellow transparency and one hundred percent yellow transparency on three skin tones.

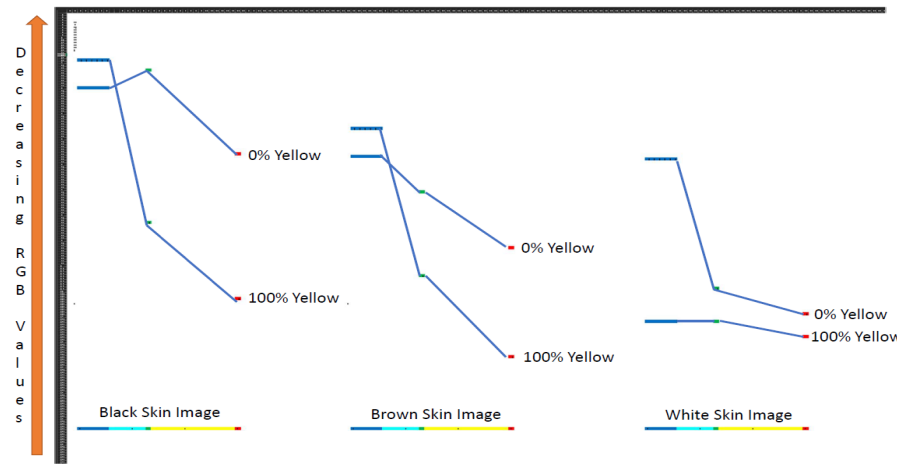

Figure 34: RGB values that correspond with zero percent and one hundred percent yellowness in three skin tones

Note that we flipped the graph by showing the lower values as you go up. In doing so, the behavior of the lines mimics the graph presented by Nabarun Polley and his team in 2015 on the absorption rate of colors in conjunctiva of infants with and without jaundice.

In summary, the color experimentation revealed the following results in Table 4 below.

Table 4: Changes of RGB values from zero to $100 \%$ yellow

\begin{tabular}{|c|c|c|}
\hline $\begin{array}{c}\text { Skin } \\
\text { Tone }\end{array}$ & $\begin{array}{c}\text { Blue Color } \\
\text { Values }\end{array}$ & $\begin{array}{c}\text { Red/Green } \\
\text { Color Values }\end{array}$ \\
\hline White & Decreased & Decreased \\
\hline Brown & Decreased & Increased \\
\hline Dark & Decreased & Increased \\
\hline
\end{tabular}

Zero and $100 \%$ yellowness show differences in scores of RGB. With white skin tones, all RGB scores went down in 100\% yellowness indicating greater reflectance of light in jaundiced skin. In both brown and dark skin tones, Red and Green values increased while Blue score decreased when yellowness moved from zero to $100 \%$. Thus, jaundiced babies with brown- and darkskin tone tend to reflect Red and Green colors more than the nonjaundiced infants.
Varied percentages of yellowness in between zero and 100\% bring about changes in the RGB scoring. Detection of smaller increments of yellowness will be further explored. We are also looking for a spectroscopic approach related to the study of Nabarun Polley and his team which will be reported soon.

\subsection{Mobile or Near Cloud for Smart Nursing Station}

The smart sensor and monitoring system of the Smart LPLS can be integrated with the Mobile or Near Cloud as part of the Smart Nursing Station for medical use cases. The main component of this Near Cloud System is the Raspberry Pi, a lowcost, credit card sized microcomputer that offers various interfaces. Raspbian, a Debian-based operating system, is burned to the micro SD card for the Raspberry Pi to function. For easy deployment, the system should be portable and have the necessary processing power for its essential functions.

Since content are cached in the Near Cloud node, the medical doctors and nurses as Mobile or Near Cloud clients can upload, download, or stream prepositioned health and medical data remotely in their own most convenient time using their Wi-Fi enabled devices. To observe security measures, administrators can identify users and set restrictions. Server-side encryption feature encrypts files stored on the server and files on remote storage. When encryption is enabled, all files are encrypted and decrypted by the software application and saved on the remote storage. Also, Wi-Fi Protected Access version 2 or WPA2, a method of securing your network with the use of the optional PreShared Key authentication, is offered by the node. Time-based one-time passwords was also enabled for the system to add another layer of access security to the system.

This technology complements the off-grid setup of the Smart LPLS as it is readily deployable, works with or without internet connection, and can be powered by using a mobile power bank or DC adapter. It is of great value in remote health facilities for patient monitoring of select few doctors who work in barrios lacking in medical equipment and supplies, as featured by Juan Alfonso Leonardia and his co-proponents [27]. Its architecture features load-sharing and can be interconnected with other Near Cloud nodes via an inner wireless mesh network using B.A.T.M.A.N-adv routing protocol.

\subsection{Improving Power Storage for Off-grid Deployment}

Phototherapy treatment usually requires exposure of the infant patient for an extended period. However, deployment of a phototherapy unit in remote areas can present issues on power supply which is either intermittent or totally off grid. To address this problem, we designed the smart LPLS to be more energyefficient, which makes provision of solar powering less costly and more feasible. We tested and compared the performance of lithium ion, motorcycle, and car batteries. The use of car and motorcycle batteries in powering a system in off grid deployment had been studied in a recent publication by Paul Cabacungan and his co-researchers [28].

In this paper, an experimentation on the discharging rate of the 12-volt lead-acid battery and 120 lithium-ion batteries (all of them with a lifespan of 3-5 years) was conducted using a seven-watt LED bulb. The lead-acid battery was connected to a 7-watt LED 
bulb while the lithium-ion battery that was uniformly configured (Li-ion UC), i.e. with uniform number of battery cells in parallel, was attached to the same bulb with converter. A lithium-ion battery that was reconfigured (Li-ion $\mathrm{RC}$ ), i.e., 45-30-45 batteries in parallel, used a charge controller instead of a converter. Results are shown in Table 5 below.

Table 5: Comparison of lead acid and lithium-ion batteries

\begin{tabular}{|l|l|l|l|l|}
\hline & Ampacity & $\begin{array}{l}\text { Volume } \\
\text { (sq.cm.) }\end{array}$ & $\begin{array}{l}\text { Weight } \\
\text { (kg.) }\end{array}$ & $\begin{array}{l}\text { Duration of } \\
\text { Discharging }\end{array}$ \\
\hline $\begin{array}{l}\text { Lead } \\
\text { Acid }\end{array}$ & $50 \mathrm{AH}$ & 8840 & 15 & 26 hours \\
\hline $\begin{array}{l}\text { Li-ion } \\
\text { UC }\end{array}$ & $40 \mathrm{AH}$ & 3500 & 3 & 5 hours \\
\hline $\begin{array}{l}\text { Li-ion } \\
\text { RC }\end{array}$ & $40 \mathrm{AH}$ & 3500 & 3 & 17.75 hours \\
\hline
\end{tabular}

Results showed that lead-acid batteries took longer to discharge compared to lithium-ion. However, lithium-ion has its advantages in remote deployment - being twice smaller and 5 times lighter. Moreover, when batteries are discharged below their manufacturer-stated end-of-life discharge voltage, cells are permanently damaged, preventing it from ever being recharged. Lithium-ion batteries require low maintenance because each cell, once damaged, can be individually replaced without having to replace all the batteries in the system.

We have also done initial power calculations for a set of 10 conventional $60 \mathrm{~cm}$ LED strips with 36 LEDs each. 3 LEDs draw 20 milliamperes at $12 \mathrm{~V}$ to consume a total of 28.8 watts, about $70 \%$ less power consumption than the T5 LED. In using LED strips, an overall power consumption is estimated to decrease from 240 watts (SPLS) to 90 watts (SPLS off-grid), which already includes power usage of the charge controller, sensor, and monitoring system.

For future innovations, another prototype with top and bottommounted phototherapy lights that will operate on DC can be explored to eliminate the power consumed by the $\mathrm{DC}$ to $\mathrm{AC}$ converter and the T5 LED tubes' internal AC to DC converters. This prototype can use LED strips with transparent outer cover, which is expected to provide greater irradiance compared to T5 LED with translucent cover that limits light intensity.

\section{Conclusion and Future Works}

We presented an improved architecture and design for the current system, making the Smart LPLS a viable option for rural and underserved areas with limited resources to treat newborn patients with jaundice. The integration of skin color monitoring aids medical doctors by providing a non-invasive method of possible detection and monitoring of jaundice condition. Several approaches are being taken as we describe how we are developing a complementary system of transparencies that doctors can use in the field with photos to determine the severity of the yellowing and will aid in monitoring the progress of the treatment. We acknowledge the limitation of the current results on skin color www.astesj.com monitoring, having tried the transparencies only on images with three specific kinds of skin color. We cannot claim a generalized conclusion yet, thus, we recommend that for future development, we can increase the sample size and cover a wider variety of skin colors. Also, aside from the usual placing of sensors on the forehead and chest, we explore putting it on other parts of the body such as nails, palm or sole.

The robust LED spotlights mounted on top and the additional T5 LED tube lights at the bottom offer more powerful and faster jaundice treatment due to greater irradiance. Its low power consumption and solar powering provision make it easily deployable even in remote off grid health systems. The Mobile Cloud System provides easy access to data which can supplement the patient's existing records.

The initial tests conducted in several patients exhibit results that are promising with treatment outcome and safety features comparable to commercial units available in the market today. However, we have yet to conduct further tests with actual human subjects, under the supervision of medical personnel to generalize results. To sum up, the Smart Low-cost Phototherapy Light System integrated with additional functions can still be made more energy-efficient, appropriately deployable in off-grid locations and yet maintain its affordable cost.

\section{Acknowledgment}

The prototypes built by Ateneo Innovation Center went under extensive research acceptance tests in public hospitals in Metro Manila by our collaborators - Dr. Vanessa Marie Calabia, Dr. Viel Bagunu, Dr. Ma. Lucila Perez, and Dr. Jeremie De Guzman. We thank them for their efforts and valuable feedback. We are grateful to our researchers, Mr. Alfonso Agustin, Mr. Alfonso Cuezon, Mr. Reymond Cao, Mr. Kerwin Caballas, Mr. Lawrence Ibarrientos, Mr. Lemuel Emman Tan and the interns and OJTs who worked in th research center for the past five years. In their capacities, they gave their valuable assistance in the different stages of fabricating the prototypes. We are indebted to the University Research Council (URC) of the Ateneo de Manila University for granting financial support to come up with this paper and to explore further developments of the Smart LPLS. Our gratitude goes to the Ateneo Innovation Center for serving as a hub for the phototherapy light system developments since its initial conceptualization.

\section{References}

[1]. P. Cabacungan, C. Oppus, J. De Guzman, G. Tangonan, I. Culaba and N Cabacungan, "Intelligent Sensors and Monitoring System for Low-cost Phototherapy Light for Jaundice Treatment," 2019 International Symposium on Multimedia and Communication Technology (ISMAC), Quezon City, Philippines, 1-6, August 2019, doi:10.1109/ISMAC.2019.8836133.

[2]. V. Calabia, M. Perez, G. Tangonan, P. Cabacungan, I. Culaba, and J. De Guzman. "Bilirubin lowering effect and safety of a prototype low cost blue light emitting diode (LED) phototherapy device in the treatment of indirect hyperbilirubinemia among healthy term infants in a tertiary government hospital: a pilot study," arXiv preprint arXiv:2008.08875, August 2020.

[3]. V. Bagunu and M. Perez, "Comparative study of Prototype Low-Cost Light Emitting Diode Phototherapy Device versus Conventional Fluorescent Phototherapy in the Treatment of Indirect Hyperbilirubinemia among Term Infants in a Tertiary Government Hospital," Unpublished, August 2018.

[4]. C. Henny-Harry and H. Trotman, "Epidemiology of neonatal jaundice at the University Hospital of the West Indies," The West Indian Medical Journal, 
61(1), 37-42, January 2012

[5]. P. Kumar, D. Chawla, and A. Deorari, "Light-emitting diode phototherapy for unconjugated hyperbilirubinemia in neonates," Cochrane Database of Systematic Reviews, 12(7969), December 2011, doi:0.1002/14651858.CD007969.pub2.

[6]. S. Moerschel, L. Cianciaruso, and L. Tracy, "A practical approach to neonatal jaundice," American Family Physician, 77(9), 1255-1262, May 2008.

[7]. M. Fargo, S. Grogan, and A. Saguil, "Evaluation of Jaundice in Adults," American Family Physician, 95(3), February 2017.

[8]. E. Gurley, A. Halder, P. Streatfield, H. Sazzad, T. Nurul Huda, M. Jahangir Hossain, and S. Luby, "Estimating the burden of maternal and neonatal deaths associated with jaundice in Bangladesh: possible role of hepatitis E infection," American Journal of Public Health, 102(12), 2248-2254, December 2012, doi:10.2105/AJPH.2012.300749.

[9]. Iowa Department of Public Health, "Managing Newborn Hyperbilirubinemia and Preventing Kernicterus Progeny,"http://www.idph.state.ia.us/hpcdp/common/pdf/perinatal_newslet ters/progeny_june2013.pdf. Accessed: August 2020

[10]. World Health Organization International, "Bilirubinometer - Core medical equipment - - Information," https://www.who.int/medical_devices/innovation/bilirubinometer.pdf. Accessed: August 2020.

[11]. PhilHealth, "Agency's Mandate And Functions," https://www.philhealth.gov.ph/about us/mandate.html\#gsc.tab=0. Accessed: August 2020.

[12]. H. Vreman, R. Wong, and D. Stevenson, "Phototherapy: current methods and future directions," Seminars Perinat, 28, 26-33, October 2004, doi: 10.1053/j.semperi.2004.09.003.

[13]. J. dela Cruz, N. Libatique and G. Tangonan, "Design of a Disaster Information System using Mobile Cloud Wireless Mesh with Delay Tolerant Network," 2019 IEEE Global Humanitarian Technology Conference (GHTC), Seattle, WA, USA, 1-8, October 2019, doi: 10.1109/GHTC46095.2019.9033450.

[14]. N. Mercado, Design and Demonstration of Near Cloud System for Digital Education and Disaster Resiliency, MS ECE thesis, Electronics, Computer and Communications Engineering, Ateneo de Manila University, Quezon City, Philippines, May 2020.

[15]. J. Mamaradlo, Design and Demonstration of a Mobile Cloud System for Smart Transportation System Use Case, MS ECE thesis, Electronics, Computer and Communications Engineering, Ateneo de Manila University, Quezon City, Philippines, May 2020.

[16]. J. Mamaradlo, N. Mercado, N. Libatique, G. Tangonan, R. Solis, V. Rodriguez, B. Dingel, C. Pineda, and C. Lopez, "University campus 5G testbed and use case deployments in the Philippines," SPIE Proceedings, Broadband Access Communication Technologies XIV, 11307, January 2020, doi:3. 10.1117/12.25499032020.

[17]. O. Onwe, O. Ezeanosike, C. Obu, O. Daniyan, and H. Amadi, "Economic cost savings with the use of total body phototherapy for the treatment of severe neonatal jaundice in Nigeria," Journal of Pediatrics and Neonatal Care, 9(6), December 2019, doi: 10.15406/jpnc.2019.09.00401.

[18]. A. Riskin, A. Tamir, A. Kugelman, M. Hemo, and D. Bader, "Is Visual Assessment of Jaundice Reliable as a Screening Tool to Detect Significant Neonatal Hyperbilirubinemia?," The Journal of Pediatrics, January 2008, doi: 10.1016/j.jpeds.2007.11.003

[19]. H. Baharuddin, M. Sulong, A. Joret, T. Rahman, and N. Ismail, "Bile Pigments Detection via IR Sensor," Proceedings of EnCon2010 3rd Engineering Conference on Advancement in Mechanical and Manufacturing for Sustainable Environment, Kuching, Sarawak, Malaysia, April 2010.

[20]. N. Polley, S. Saha, S. Singh, A. Adhikari, S. Das, B. Choudhury, and S. Pal, "Development and optimization of a noncontact optical device for online monitoring of jaundice in human subjects," Journal of Biomedical Optics, 20(6), June 2015, doi: 067001

[21]. Learn Leight Cotnoir, "Hue, Value, Saturation," http://learn.leighcotnoir.com/artspeak/elements-color/hue-value-saturation/. Accessed: August 2020

[22]. P. Mollica, Color Theory: An essential guide to color-from basic principles to practical applications (Artist's Library), Walter Foster Publishing, 17, January 2013.

[23]. Random Nerd Tutorials, "Guide for TCS230/TCS3200 Color Sensor with Arduino," https://randomnerdtutorials.com/arduino-color-sensor-tcs230tcs3200/. Accessed: August 2020.
[24]. Renesas Electronics, "ISL29125 | Light-to-Digital Sensors," https://www.renesas.com/us/en/products/sensors/ambient-lightsensors/light-to-digital-sensors/device/ISL29125.html. Accessed: August 2020.

[25]. Pexels.com, "Photo of Couple with Baby Sitting," https://www.pexels.com/photo/photo-of-couple-with-a-baby-sitting3995914/. Accessed: August 2020.

[26]. G. Chimhini, S. Chimhuya, and V. Chikwasha, "Evaluation of transcutaneous bilirubinometer (DRAEGER JM 103) use in Zimbabwean newborn babies," Maternal Health, Neonatology and Perinatology, 4(1) April 2018.

[27]. J. Leonardia, H. Prytherch, K. Ronquillo, R. Nodora and A. Ruppel, "Assessment of factors influencing retention in the Philippine National Rural Physician Deployment Program," BMC Health Services Research, 12(411), November 2012.

[28]. P. Cabacungan, G. Tangonan, and N. Cabacungan, "Water - Electricity Light System (WELS): Technology Innovations," 2020 International Journal of Recent Technology and Engineering (IJRTE), 8(6), 3061-3068, March 2020, doi: 10.35940/ijrte.F8103.038620 